\title{
Influenza A Virus Infection Alters Gut Microbiota Composition in Juvenile Mice
}

\author{
Eva Fuglsang1*, Lukasz Krych², Dennis S Nielsen², Hanne Frøkiær ${ }^{1}$ \\ and Patrick C Reading 3,4 \\ ${ }^{1}$ Department of Veterinary and Animal Sciences, University of Copenhagen, Denmark \\ ${ }^{2}$ Department of Food Science, University of Copenhagen, Denmark
}

\section{Research Article \\ Volume 2 Issue 3}

Received Date: November 05, 2018

Published Date: November 13, 2018

${ }^{3}$ Department of Microbiology and Immunology, University of Melbourne, at the Peter Doherty Institute for Infection and Immunity, Australia

4WHO Collaborating Centre for Reference and Research on Influenza, at the Peter Doherty Institute for Infection and Immunity, Australia

*Corresponding author: Eva Fuglsang, Department of Veterinary and Animal Sciences, University of Copenhagen, Frederiksberg, Denmark; Email: fuglsangeva@gmail.com

\section{Abstract}

The gut microbiota (GM) stimulates the immune system to facilitate appropriate immune responses at local and distal sites of virus infection. However, the impact of distal virus infections in modulating the composition of GM is less clear. Here, a mouse model was used to examine the effect of influenza a virus (IAV) infection on the composition of GM in mice that did or did not receive oral treatment with broad spectrum antibiotics (ABX). We focused on ABX treatment of 3 weeks old juvenile mice, as this represents a dynamic time in the establishment of GM and immunity, followed by mockor IAV-infection 2 weeks later. Mice were subsequently assessed for changes in body weight, viral load in the respiratory tract and the composition of GM. As anticipated, oral ABX treatment reduced the diversity of GM and ABX-treated mice showed exacerbated disease, characterized by enhanced weight loss and increased virus titers in the upper and lower airways. Surprisingly, IAV infection also increased the diversity of GM in ABX-treated mice, resulting in a microbial composition qualitatively and quantitatively different to mock-infected ABX-treated mice and IAV-infected mice that did not receive ABX treatment. Specifically, IAV infection increased the relative abundance of eight different bacteria in GM in ABX mice compared to the GM in mock-infected ABX mice. These findings confirm an important role for GM in functional immune defense against IAV infection in juvenile mice, consistent with previous studies in adult animals. Moreover, we report for the first time that distal IAV infection of the airways can enhance the diversity of an ABX-compromised GM.

Keywords: Gut Microbiota; Antibiotic; Influenza A Virus; Mouse Model; Akkermansia Muciniphila 


\section{Journal of Infectious Diseases \& Travel Medicine}

Abbreviations: ABX: broad-spectrum antibiotic; CON: control; i.n: intranasal; GM: Gut microbiota; i.t: intratracheal; IAV: influenza A virus; MDCK: Madin-Darby canine kidney; NMDS: Non-metric multidimensional scaling; OUT: Operational Taxonomic Units; p.i: postinfection; PFU: plaque forming units; QIIME: Quantitative Insight In to Microbial Ecology.

\section{Introduction}

Gut microbiota (GM) dysbiosis plays an important role in the etiology of an array of diseases, including resistance towards infections caused by Antharam, et al. [1], Koenigsknecht, et al. [2], Schubert [3], Zainah, et al. [4], parasites e.g. Toxoplasma gondii [5] and viruses e.g. influenza A virus (IAV) [6-9], and human immunodeficiency virus (HIV)-1 [10]. A homeostatic GM can contribute to protection against invading pathogens via depletion of nutrients, antimicrobial peptide production and/or priming of immune cells to combat infections at local or distal sites in the body $[11,12]$. Exposure to oral antibiotics (ABX) can alter GM diversity and this can differ depending on the particular ABX treatment [6]. In mouse models, reduced GM diversity during bacterial or viral challenge is known to exacerbate diseases e verify [2,3,6-8,13-15]. Modulation of GM following pre-treatment of adult mice with ABX resulted in exacerbated disease following subsequent IAV challenge, characterized by delayed virus clearance, reduced titers of virus-specific antibodies and impaired inflamma some-dependent responses by innate and adaptive immune cells [6,7]. Thus, commensal microbes appear to play a critical role in calibrating appropriate immune responses to IAV and other pathogens.

Colonization of the gastrointestinal tract by microorganisms commences at birth, immediately after delivery, and the succession of new bacterial colonizers have been shown to be essential for development of the immune system [16-18]. Not surprisingly, reduced microbiota diversity as a result of ABX treatment or germfree conditions in early life can affect development of the immune system [19-21]. Making the host more vulnerable to infection. In mice, establishment of intestinal microbiota occurs after weaning between $3-5$ weeks postpartum [22]. While the impact of ABX treatment on IAV infection has been established in adult mice, we have investigated the impact of $\mathrm{ABX}$ treatment of juvenile animals ( 3 weeks old) given that this time is critical for the final establishment of GM composition.

Following intranasal (i.n.) or intra tracheal (i.t.) inoculation of mice with seasonal IAV, epithelial cells lining the upper and lower respiratory tract represent the primary targets for IAV infection and amplification. The expression of particular trypsin-like proteases in the airways is a major factor limiting the systemic spread of seasonal IAV to other sites in the body. Consistent with this, infectious virus is generally not recovered from the intestinal tractor other distal organs $[23,24]$. Nevertheless, experimental infection of mice by the mouse-adapted IAV strain A/Puerto Rico/8/1934 (PR8, (H1N1)) altered GM composition and was associated with increase dintestinal injury $[9,24]$. Furthermore, one of these studies found that use of broad-spectrum ABX prior to and during PR8 infection diminished infection-related intestinal injury. Together, these studies are consistent with an indirect effect of IAV infection on the composition of GM and/or intestinal health in adult mice [9].

Strains of human seasonal IAV show marked differences in their ability to infect and induce disease in mice. Historically, serial passage of human IAV in mice has been used to increase their virulence, allowing for studies investigating pathogen city and immune responses elicited to IAV infection. PR8 is a mouse-adapted H1N1 virus that has been widely used to study IAV infection in laboratory mice as it readily infects the lung to induce a viral pneumonia, similar to that seen during severe disease in humans [25]. However, primary viral pneumonia represents the most severe pulmonary manifestation and is relatively uncommon in healthy adults during most seasonal epidemics [26,27]. Moreover, the virulence of the PR8 strain in mice can limit its usefulness, particularly in immune compromised animals or in juvenile mice which also show increased susceptibility to IAV [28]. Herein, we have investigated the ability of HKx31 (H3N2), an IAV strain of moderate virulence in mice compared to PR8 [29,30]. To infect and modulate the composition of GM in juvenile mice. In addition, we have examined the effect of oral ABX treatment prior to and during $\mathrm{HKx} 31$ infection on the composition of GM in juvenile mice.

\section{Materials and Methods}

\section{Ethics Statement}

All experiments with mice were conducted with approval from the University of Melbourne Biochemistry \& Molecular Biology, Dental Science, Medicine, Microbiology \& Immunology, and Surgery Animal Ethics Committee. Experiments using mice were conducted with approval from the University of Melbourne Biochemistry \& Molecular Biology, Dental Science, Medicine, Microbiology \& Immunology, and Surgery Animal Ethics Committee (project 1413227.3), in accordance with the National Health and Medical Research Council (NHMRC) 


\section{Journal of Infectious Diseases \& Travel Medicine}

Australian code of practice for the care and use of animals for scientific purposes.

Experiments using 10-day embryonated chicken eggs were conducted with approval from the University of Melbourne Biochemistry \& Molecular Biology, Dental Science, Medicine, Microbiology \& Immunology, and Surgery Animal Ethics Committee (project 1714213), in accordance with the National Health and Medical Research Council (NHMRC) Australian code of practice for the care and use of animals for scientific purposes. Eggs were obtained from Hy-Line Australia (Bags hot, Victoria, Australia).

\section{Mice and Virus}

C57BL/6 male mice were bred and housed in specific pathogen-free facilities at the Department of Microbiology and Immunology, University of Melbourne, Australia. IAV strain $\mathrm{HKx} 31$ is a high-yielding reassortant of $\mathrm{A} / \mathrm{PR} / 8 / 34$ (PR8, H1N1) and A/Aichi/2/1968 (H3N2), and expresses the H3N2 surface glycoproteins. HKx31 was grown in 10day embryonated hen's eggs by standard procedures [31]. Viral stock was stored at $-80^{\circ} \mathrm{C}$. Titers of infectious virus were determined by standard plaque assay on MadinDarby canine kidney (MDCK, American Type Culture Collection (ATCC)) cells [31]. Expressed as plaqueforming units (PFU)/ml.

\section{Antibiotic Treatment and IAV Infection of Mice}

Following weaning at 3 weeks of age, mice received either (i) normal drinking water, or (ii) drinking water supplemented with ampicillin $(0.5 \mathrm{mg} / \mathrm{ml})$, gentamicin sulphate $(0.5 \mathrm{mg} / \mathrm{ml})$, and metronidazole $(0.5 \mathrm{mg} / \mathrm{ml})$ (all from Sigma Aldrich, collectively called ABX). Mice were treated for 3 weeks and ABX water was changed every third day. After 2 weeks $\pm A B X$ treatment (i.e. 5 weeks of age), mice were anaesthetized using isoflurane and infected via the intranasal (i.n.) route with $100 \mathrm{PFU}$ of HKx31 in 50 $\mu$ PBS. Control mice were mock-infected with $50 \mu \mathrm{l}$ of PBS alone. Mice were weighed daily and assessed for signs of clinical disease. Animals that had lost $>15 \%$ of their original body weight and/or displayed evidence of pneumonia were culled. On day 7 p.i., mice were culled and nasal and lung tissues collected from IAV-infected mice were homogenized in $1 \mathrm{ml}$ of PBS, clarified by centrifugation and stored at $-80^{\circ} \mathrm{C}$. Titers of infectious virus were determined by plaque assay MDCK cells [31]. All experiments with mice were conducted with approval from the University of Melbourne Biochemistry \& Molecular Biology, Dental Science, Medicine, Microbiology \& Immunology, and Surgery Animal Ethics Committee.

\section{Fecal Sample Collection and Microbial DNA Extraction}

Fecal samples were collected from mice on the day of infection (day 0 p.i., mice $=5$ weeks of age) and immediately prior to euthanasia (day 7 p.i., mice $=6$ weeks of age) and stored at $-80^{\circ} \mathrm{C}$ until DNA extraction. Fecal samples were homogenized and DNA was extracted via MoBio Power Soil Kit (Mo Bio Laboratories, QIAGEN) and stored at $-80^{\circ} \mathrm{C}$ prior to analysis.

\section{High Throughput Sequencing of the Gut Microbiota}

Fecal microbiota composition of 38 mice was determined using tag-encoded 16S rRNA gene MiSeqbased (Illumina, CA, USA) high throughput sequencing. Cellular DNA extraction, DNA storage and sequencing library preparation steps were conducted as previously described [32]. The following adjustments: the V3 region of the 16S rRNA gene amplified using primers compatible with Nextera Index Kit (Illumina; NXt_338_F: 5'TCGTCGGCAGCGTC AGATGTGTATAAGAGACAG ACWCCTACGGGWGGCAGCAG-3' and NXt_518_R: 5'GTCTCGTGGGCTCGG AGATGTGTATAAGAGACAG ATTACCGCGGCTGCTGG-3'). Two samples were excluded from the analysis due to a low read number $(<10.000$ reads).

The raw data set containing pair-ended reads with corresponding quality scores were merged and trimmed using fastq_mergepairs and fastq_filter scripts implemented in the UPARSE pipeline [33]. The minimum overlap length of trimmed reads (150 bp) was set to 100 $\mathrm{bp}$. The minimum length of merged reads was $150 \mathrm{bp}$. The maximum expected error $\mathrm{E}=2.0$, and first truncating position with quality score $\mathrm{N} \leq 4$. Purging the data set from chimeric reads and constructing de novo operational taxonomic units (OTU) were conducted using the UPARSE pipeline [33]. Quantitative Insight into Microbial Ecology (QIIME) open source software package [34] (1.7.0 and 1.8.0) was used for subsequent analysis steps. The green genes (13.8) 16S rRNA gene collection was used as a reference database [35].

Unweighted, generalized, and weighted UniFrac distance metrics were calculated from subsampled OTU tables (10.000 reads/sample) and visualized with nonmetric multidimensional scaling (NMDS) plots. The differences in ordination between categories were tested using Per mutational Multivariate Analysis of Variance using Distance Matrices, 'Adonis' function from R package 'vegan' [36]. 


\section{Journal of Infectious Diseases \& Travel Medicine}

Alpha diversity measures expressed with an observed species (sequence similarity 97\% OTUs) value were computed for rarefied OTU tables (10.000 reads/sample) using the alpha rarefaction workflow. Differences in alpha diversity were determined using a t-test-based approach employing the non-parametric (Monte Carlo) method (999 permutations) implemented in the compare alpha diversity workflow. The differences in taxa abundance between categories were estimated with a statistic framework: analysis of composition of microbes (ANCOM) based on non-normalized species level OTU-table [37].

\section{Results}

\section{Oral ABX Treatment Enhances IAV-Associated Disease in Juvenile Mice}

Following weaning at 3 weeks of age, mice received normal drinking water (control (CON)) or drinking water supplemented with broad-spectrum antibiotics (ABX, gentamicin, ampicillin, and metronidazole) for 2 weeks.
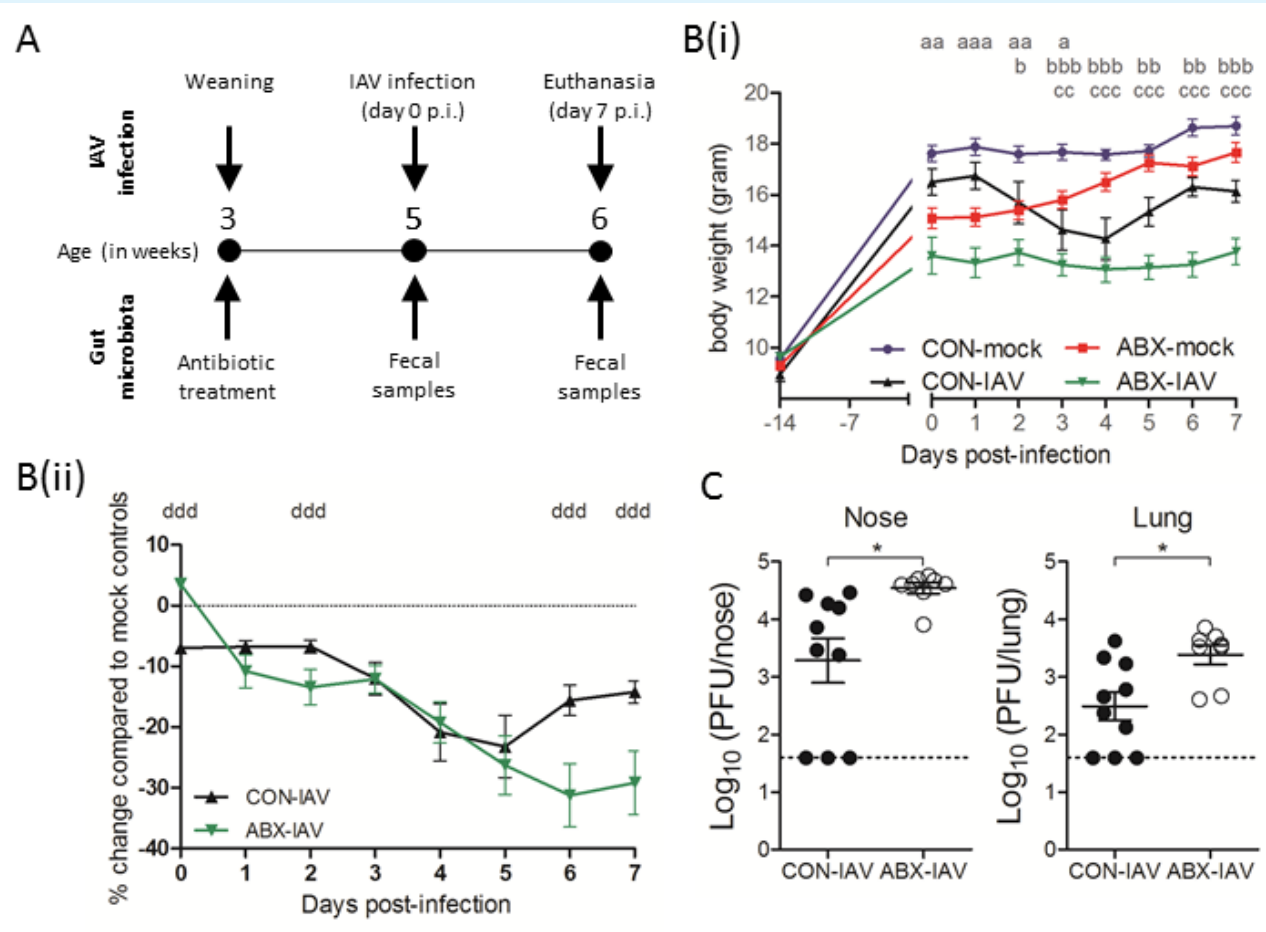

Figure 1: Oral broad-spectrum ABX treatment prior to and during IAV infection enhances IAV-induced disease in juvenile mice. (A) Experimental model. Following weaning of male mice at 3 weeks of age, half were immediately given drinking water supplemented with broad-spectrum antibiotics (ABX: ampicillin, gentamycin and metronidazole, $0.5 \mathrm{mg} / \mathrm{ml}$ ) for 2 weeks and half of the mice received normal drinking water alone (control (CON)). At 5 weeks of age, half of the ABX and CON mice were intranasally infected with 100 PFU HKx31 (ABX-IAV and CON-IAV mice, respectively) and half were mock-infected with virus diluent (ABX-mock and CON-mock mice, respectively). $A B X$ were maintained in the drinking water of ABX-mock and ABX-IAV mice throughout the course of infection (7 days). All mice were euthanized for analysis at day 7 post-infection (p.i.). Fecal samples were collected for analysis of microbiota at day 0 and day 7 p.i. (B) Body weight changes measured as (i) grams, (ii) percentage of original body weight on day 0 relative to infection (CON-mock, blue, $n=10$; CON-IAV, black, $n=10$; ABX-mock, red, $n=10$; ABX-IAV, green, $n=8$ ), and (iii) percentage weight change relative to appropriate mock-infected controls (based on body weight in grams; CON-IAV, open black; ABX-IAV, open green). Data show the mean \pm SEM for groups of 10 mice, pooled from two independent experiments and were analyzed by two-way ANOVA followed by Bonferroni's post-test. $\mathrm{a}=\mathrm{CON}$-mock vs. ABX-mock, $b=$ CON-mock vs. CON-IAV, $c=A B X-m o c k$ vs. ABX-IAV, $d=$ CON-IAV vs. ABX-IAV where $p<0.001$, $\mathrm{p}<0.01$ and $\mathrm{p}<0.05$ are denoted by 3, 2 or 1 letters, respectively.(C) Virus titers were determined in clarified homogenates prepared from lung and nasal tissues of IAV-infected mice. Titers from individual animals are shown and horizontal bars represent the mean \pm SD for each group. The dashed line shows the detection limit of the assay. Data are pooled from two independent experiments. Data were analyzed using Student's t-test. *p<0.05. 
After this time, mice were infected via the intranasal (i.n.) route with 100plaque-forming units (PFU) of IAV strain HKx31 (IAV) or mock infected with an equivalent volume of virus diluent (mock). IAV- and mock-infected mice were monitored for a further 7 days with ABX present or absent from the drinking water (for experimental design (Figure 1A). Mice were weighed daily after commencement of ABX treatment and following IAV infection. Note that no clinical signs of disease were evident throughout the monitoring period (data not shown). Compared to CON mice, two weeks of $A B X$ treatment resulted in a significant weight reduction on the day of infection (Figure 1B(i)), day -14 to 0 postinfection (p.i.). These findings are consistent with previous studies showing that ABX treatment of juvenile mice is associated with delayed weight gain [38]. Next, we assessed the impact of IAV infection on weight loss in ABX- and CON-treated mice by examining body weight in grams (Figure $1 \mathrm{~B}(\mathrm{i})$ ) or as the percentage change in each of the IAV-infected groups relative to their appropriate mock-infected control (Figure 1B (ii)). The mock-infected mice, CON-mock animals showed minimal weight gains between day 0-7 p.i. whereas ABX-mock mice continued to gain weight during this time (Figure 1B (i)). These findings are consistent with studies showing that ABX treatment of adult mice is associated with increased weight gain $[39,40]$. Interestingly, when examining the percentage weight change compared to the appropriate mock-infected control, it was evident that maximum relative weight loss for CON-IAV animals occurred at days 4-5 p.i., whereas ABX-IAV continued to lose weight and maximum relative weight loss was recorded at days6-7 p.i. (Figure 1C (ii)). While IAV infection of both CON and $\mathrm{ABX}$ mice induced significant weight loss compared to respective mock-infected animals, viral replication was enhanced in both the upper (nose) and lower (lungs) respiratory tract of ABX-IAV mice compared to CON-IAV mice (Figure 1D). Thus, while HKx31 infection of juvenile CON mice was associated with weight loss and viral replication in the airways, ABX treatment prior to and during infection resulted in prolonged weight loss (indicative of disease) and enhanced virus replication.

\section{Parabacteroides Distasonis was the Dominating Taxa after Oral Antibiotic Treatment at the Time of IAV Infection}

To determine the effects of $\mathrm{ABX}$ treatment on GM composition at the time of IAV infection, we examined the microbial composition in fecal samples collected on the day of infection (i.e. day 0 p.i., after two weeks of ABX treatment) and displayed all tax a with an abundance threshold of $0.1 \%$. Compared to CON mice, ABX mice showed an overall reduction in the relative abundance of most types of bacteria and the GM of ABX mice was dominated by Parabacteroides distasonis (Bacteroidetes) at levels of $87.5 \%$ in $\mathrm{ABX}$ mice compared to $7.4 \%$ in $\mathrm{CON}$ mice (Figure 2).

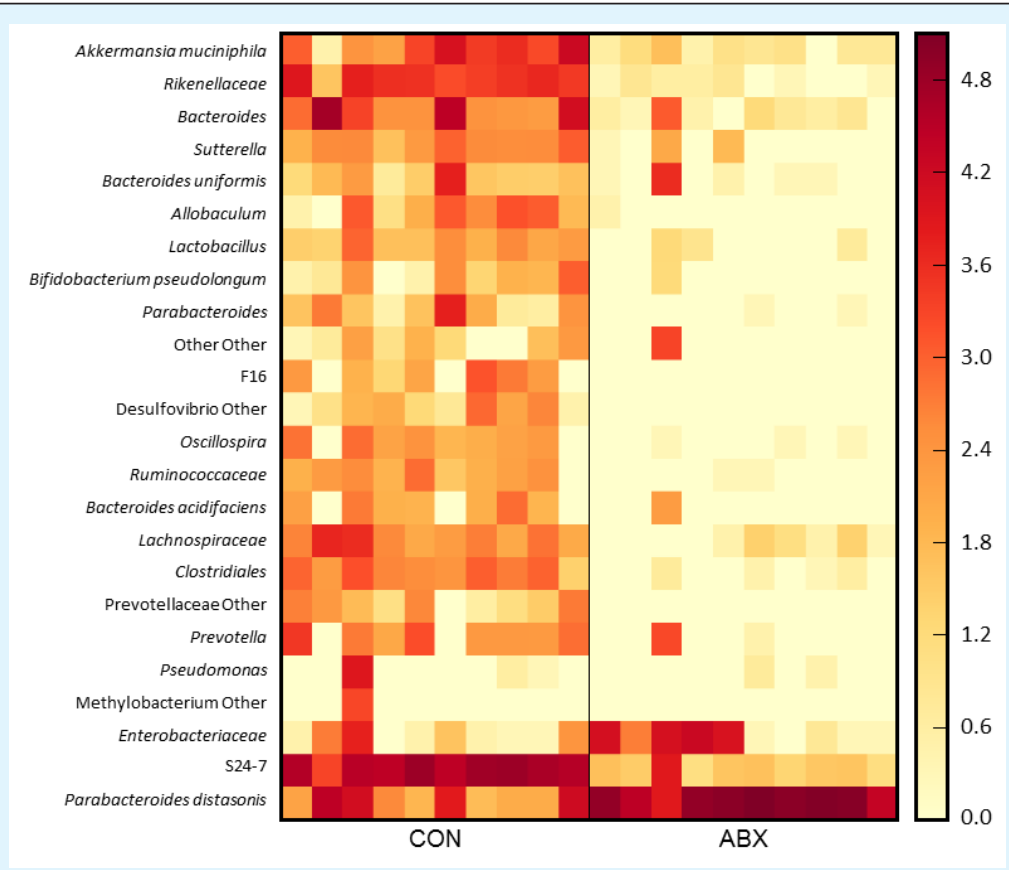

Figure 2: Oral broad-spectrum ABX treatment changes gut microbiota diversity in juvenile mice just prior to IAV infection. 
Heat map depicting taxa relative abundance reported as significantly different $(\mathrm{ANCOM}, \mathrm{p}<0.05)$ between CON $(\mathrm{n}=10)$ and $\operatorname{ABX}(\mathrm{n}=10)$ mice on day 0 p.i., just prior to IAV infection. Analyses were performed using nonnormalized, summarized to the species level OTU-table with abundance threshold $0.1 \%$. Instead, members of S24-7 represented the dominant phyla detected in the GM of $\mathrm{CON}$ mice at $58.1 \%$ compared to just $2 \%$ in $\mathrm{ABX}$ mice. The decrease in relative abundance of most bacteria was paralleled by a strong reduction in the measurement of observed species, showing a marked reduction in the diversity of GM in ABX mice compared to CON mice.

\section{IAV Infection Modulates the Composition of Gut Microbiota in ABX Mice}

The non-metric multidimensional scaling (NMDS) plots based on unweighted, generalized and weighted UniFrac distance matrices showed significant (Adonis $\mathrm{p}<0.01$ ) separation between the 4 groups, indicating clear qualitative and quantitative differences in microbial composition (Figure 3).
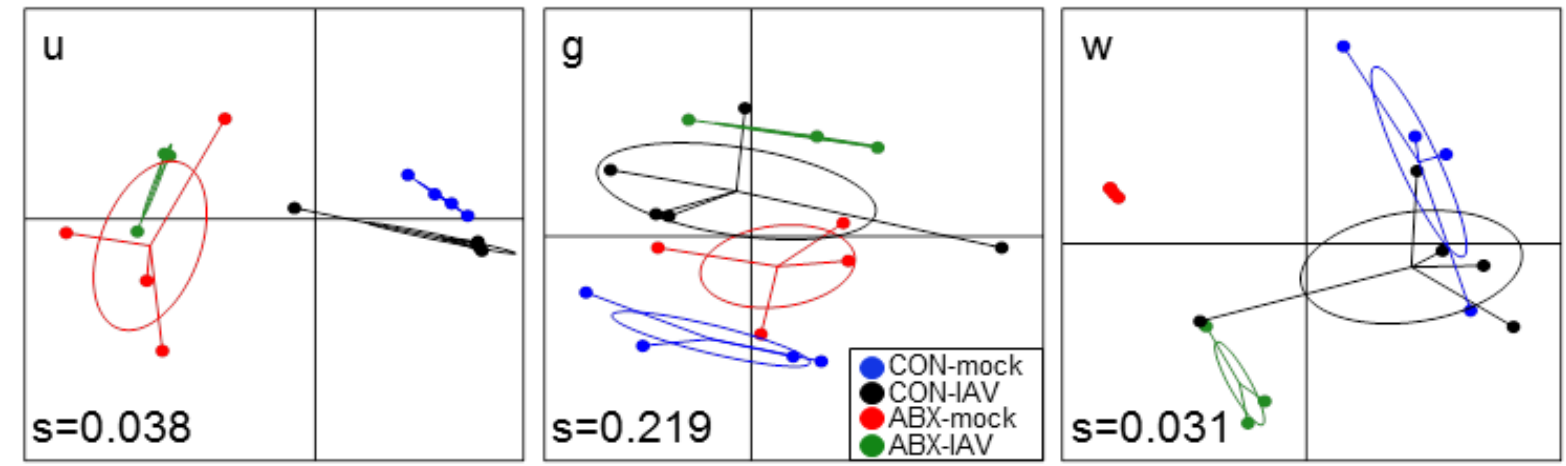

Figure 3: IAV infection enhances the diversity of the compromised gut microbiota in ABX mice. Nonmetric multidirectional scaling (NMDS) plots based on unweighted $(\mathrm{u})$, generalized $(\mathrm{g})$, and weighted $(\mathrm{w})$ UniFrac distance matrices. Clear separation between all 4 treatment groups on unweighted (adonis R2 $=0.593 ; p<0.01^{* *}$ ) and generalized (adonis R2 $=0.675 ; \mathrm{p}<0.01^{* *}$ ). NMDS plots indicate that main differences are to be expected within low and semi-abundant taxa. The NMDS plot based on the weighted UniFrac distance matrix indicates differences in high abundant taxonomic group within ABX-IAV group (adonis R2 $\left.=0.687 ; \mathrm{p}<0.01^{* *}\right)(\mathrm{w})$. S values represent the stress of each NMDS ordination. (CON-mock (blue) $n=4, \operatorname{CON}-\mathrm{IAV}$ (black) $n=5, A B X-$ mock (red) $n=4, A B X-I A V$ (green) $n=3$ ).

The bacterial diversity (observed species index) tended to increase in both of the infected groups (CONIAV and ABX-IAV), however this was only significant between ABX-mock and ABX-IAV mice (Figure 4A). Infection of CON mice (CON-IAV) significantly reduced the relative abundance of Allobaculum (Firmicutes) (Figure 4B). Interestingly, infection of $\mathrm{ABX}$ mice resulted in a significant increase in relative abundance of severaltaxa, including Akkermansia muciniphila (Verrucomicrobia), Enterobacteriaceae and Sutterella (Proteobacteria),
Bacteroides, Candidate phyla S24-7, Bacteroides uniformis, Bacteroides acidifaciens and Bacteroidales (all Bacteroidetes), (Figure 4C).

While the number of animals, and hence fecal samples analyzed in the present study was relatively low, it was sufficient to provide statically confidence in differences in the diversity of GM. No doubt that the quality and statistical power of this data would be improved by additional sampling. 


\section{Journal of Infectious Diseases \& Travel Medicine}

A

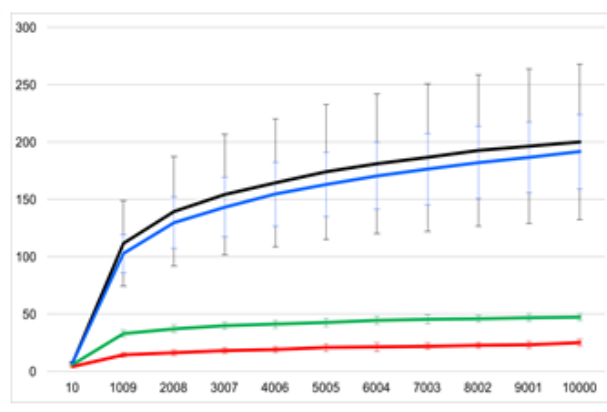

C

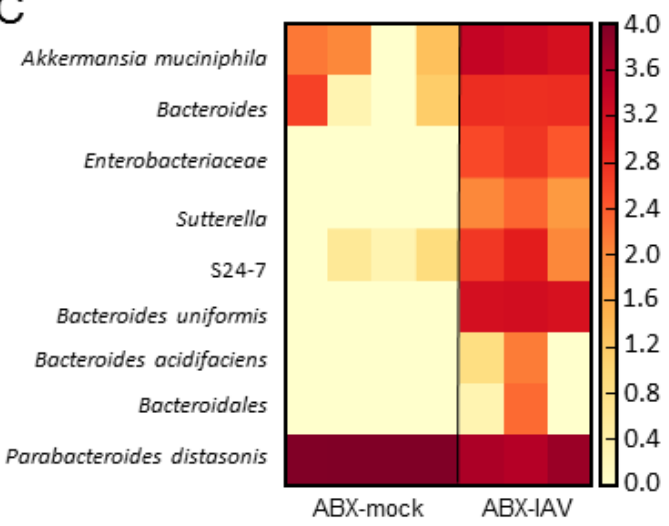

B

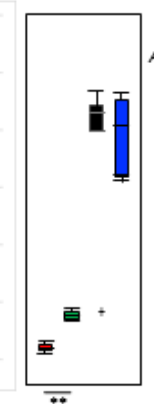

Akkermansia muciniphila

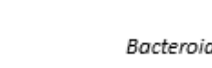

Oscillospira

Prevotellaceae

Ruminococcaceae

[Prevotella]

Lachnospiraceae

Odoribacter

Prevotella Other

Ruminococcus

Clostridiales

Bacteroides acidifaciens

Bacteroidales

Sutterella

Allobaculum *

Enterobacteriaceae

S24-7

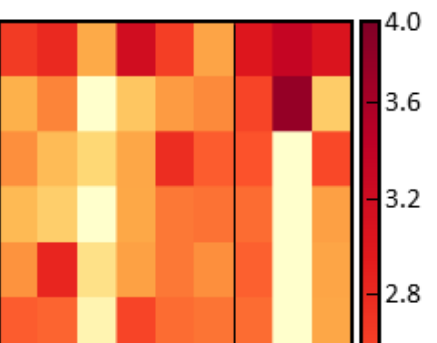

2.8

2.4
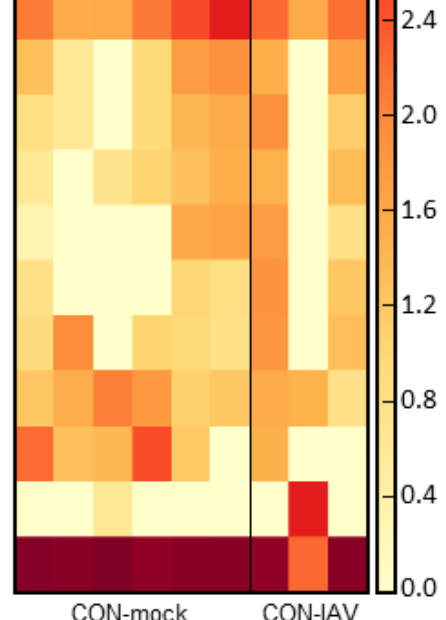

\section{.2}

0

3.6

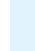

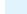


the immune system, which must adjust from GM maintenance towards limiting IAV infection thereby facilitating uncontrolled growth of Parabacteroides distasonis. Thus, our studies suggest that in the context of ABX treatment, a moderate IAV infection represents an additional factor that may modulate GM diversity in a juvenile population.

Two studies reported that IAV infection of adult SPF mice induced changes in the GM composition $[9,24]$. This was associated with increased susceptibility to secondary bacterial infections [24]. In these studies, infection of adult mice (6-10 weeks of age) with sub-lethal doses of PR8 resulted in weight loss, disease and immunopathological lung injury $[9,24]$. PR8 infection was also associated with recruitment of T helper 17 (Th17) cells and local interferon- $\gamma$ production to modulate the intestinal microbiota (including an increase in Enterobacteriaceae), thereby contributing to intestinal injury [9]. Furthermore, eradicating most of the GM with a broad spectrum ABX or specifically targeting Escherichia coli with streptomycin prior to PR8 infection reduced pathological injury of the small intestine [9]. In the present study, infection of juvenile mice with HKx31 did not affect the relative abundance of Enterobacteriaceae in CON-IAV mice compared to CON-mock mice, despite clear evidence of IAV-induced disease (as evidenced by virus replication and weight loss over time). However, ABX treatment of mock-infected animals increased the relative abundance of Enterobacteriaceae in the gut, which was further increased in ABX-IAV mice. Thus, HKx31 infection of juvenile mice does not exert the same indirect immune modulatory effects as PR8 infection of adult mice with regard to Enterobacteriaceae. Nonetheless, the combination of ABX-treatment and HKx31infection did increase the relative abundance of Enterobacteriaceae. In future studies, it will be of interest to determine if the abundance of Enterobacteriaceae observed in ABX-IAV mice is also associated with enhanced intestinal injury in these animals.

In our study, $\mathrm{ABX}$ treatment alone reduced overall GM diversity while $\mathrm{HKx} 31$ infection of $\mathrm{ABX}$-treated mice resulted in increased diversity, specifically in regard to the relative abundance of eight different bacteria. Five of these belonged to the phylum of Bacteroidetes (Bacteroides, Bacteroides uniformis, Bacteroides acidifaciens, Bacteroidales and Candidate phyla S24-7), as well as two Proteobacteria (Sutterella and Enterobacteriaceae) and one Verrucomicrobia (Akkermansia muciniphila). Akkermansia muciniphila uses gastrointestinal mucin as its carbon and nitrogen source [41]. The increased presence in ABX-IAV mice suggests that viral infection may promote its growth, possibly via enhancing mucin production in the gut. This appears plausible since (i) it is established that IAV infection in the airways can induce immunomodulatory effects in the intestines and (ii) that a range of bioactive molecules, including cytokines and growth factors, can modulate mucin secretion in the gut [42]. In future studies, it would be of interest to investigate the relationship between growth of Akkermansia muciniphila and the ability of IAV infection to induce mucin secretion in the gut of ABXtreated and control animals.

Despite the increase in GM diversity between ABX-IAV and $A B X-m o c k$ animals, $A B X$ treatment prior to and during IAV infection was associated with exacerbated weight loss as well as enhanced virus replication in the airways. In future studies it will be of interest to investigate mechanisms underlying the ability of a moderate IAV infection to promote enhanced growth of specific gut bacteria in ABX-compromised mice. Further, it will be important to clarify the role of the enhanced GM diversity as it did not ameliorate disease severity in IAVinfected animals. Whether IAV-induced changes in ABXcompromised GM play any role in modulating disease severity should be the focus of future studies.

\section{Acknowledgment}

We thank Doris Frances Mikic for excellent technical assistance to this project.

\section{Author Contributions Statement}

EF, HF and PCR designed research; EF, LK and PCR performed research, EF and LK analyzed data, and EF, LK, DSN, HF and PCR wrote the paper.

\section{Funding}

This work was supported by Project Grant APP1044976 from the National Health and Medical Research Council (NHMRC) of Australia. The Melbourne WHO Collaborating Centre for Reference and Research on Influenza is supported by the Australian Government Department of Health. The project was also supported by the Danish Strategic Research Council (NEOMUNE, grant number 12-132401) and the Augustinus Foundation.

\section{Conflict of Interest Statement}

The authors declare no conflict of interest.

\section{References}

1. Antharam VC, Li EC, Ishmael A, Sharma A, Mai V, et al. (2013) Intestinal dysbiosis and depletion of 


\section{Journal of Infectious Diseases \& Travel Medicine}

butyrogenic bacteria in Clostridium difficile infection and nosocomial diarrhea. J Clin Microbiol 51(9): 2884-2892.

2. Koenigsknecht MJ, Theriot CM, Bergin IL, Schumacher CA, Schloss PD, et al. (2015) Dynamics and establishment of Clostridium difficile infection in the murine gastrointestinal tract. Infect Immun 83(3): 934-941.

3. Schubert AM, Sinani H, Schloss PD (2015) AntibioticInduced Alterations of the Murine Gut Microbiota and Subsequent Effects on Colonization Resistance against Clostridium difficile. MBio 6(4): e00974.

4. Zainah H, Hassan M, Shiekh-Sroujieh L, Hassan S, Alangaden G, et al. (2015) Intestinal microbiota transplantation, a simple and effective treatment for severe and refractory Clostridium difficile infection. Dig Dis Sci 60(1): 181-185.

5. Benson A, Pifer R, Behrendt CL, Hooper LV, Yarovinsky F (2009) Gut commensal bacteria direct a protective immune response against Toxoplasma gondii. Cell Host Microbe 6(2): 187-196.

6. Ichinohe T, Pang IK, Kumamoto Y, Peaper DR, Ho JH, et al. (2011) Microbiota regulates immune defense against respiratory tract influenza A virus infection. Proceedings of the National Academy of Sciences of the United States of America 108(13): 5354-5359.

7. Abt MC, Osborne LC, Monticelli LA, Doering TA, Alenghat T, et al. (2012) Commensal Bacteria Calibrate the Activation Threshold of Innate Antiviral Immunity. Immunity 37(1): 158-170.

8. Wu S, Jiang ZY, Sun YF, Yu B, Chen J, et al. (2013) Microbiota Regulates the TLR7 Signaling Pathway Against Respiratory Tract Influenza A Virus Infection. Current Microbiology 67(4): 414-422.

9. Wang J, Li F, Wei H, Lian ZX, Sun R, et al. (2014) Respiratory influenza virus infection induces intestinal immune injury via microbiota-mediated Th17 cell-dependent inflammation. Journal of Experimental Medicine 211(12): 2397-2410.

10. Pérez-Santiago J, Gianella S, Massanella M, Spina CA, Karris MY, et al. (2013) Gut Lactobacillales is associated with higher CD4 and less microbial translocation during HIV infection. AIDS 27 (12): 1921-1931.
11. Kamada N, Chen GY, Inohara N, Núñez G (2013) Control of pathogens and pathobionts by the gut microbiota. Nat Immunol 14(7): 685-690.

12. Zhang N, He QS (2015) Commensal Microbiome Promotes Resistance to Local and Systemic Infections. Chin Med J (Engl) 128(16): 2250-2255.

13. Ganal SC, Sanos SL, Kallfass C, Oberle K, Johner C, et al. (2012) Priming of natural killer cells by nonmucosal mononuclear phagocytes requires instructive signals from commensal microbiota. Immunity 37(1): 171186.

14. Wang J, Li F, Sun R, Gao X, Wei H, et al. (2013). Bacterial colonization dampens influenza-mediated acute lung injury via induction of M2 alveolar macrophages. Nat Commun 4: 2106.

15. Fuglsang E, Krych L, Lundsager MT, Nielsen DS, Frøkiaer H (2018) Postnatal Administration of Lactobacillus rhamnosus HN001 Ameliorates Perinatal Broad-Spectrum Antibiotic-Induced Reduction in Myelopoiesis and T Cell Activation in Mouse Pups. Mol Nutr Food Res e1800510.

16. Hill DA, Artis D (2010) Intestinal bacteria and the regulation of immune cell homeostasis. Annu Rev Immunol 28: 623-667.

17. Reading NC, Kasper DL (2011) The starting lineup: key microbial players in intestinal immunity and homeostasis. Front Microbio 2: 148.

18. Chung H, Pamp SJ, Hill JA, Surana NK, Edelman SM, et al. (2012) Gut immune maturation depends on colonization with a host-specific microbiota. Cell 149(7): 1578-1593.

19. Hansen CH, Nielsen DS, Kverka M, Zakostelska Z, Klimesova K, et al. (2012) Patterns of Early Gut Colonization Shape Future Immune Responses of the Host. Plos One 7(3): e34043.

20. Zeissig S, Blumberg RS (2014) Life at the beginning: perturbation of the microbiota by antibiotics in early life and its role in health and disease. Nature Immunology 15(4): 307-310.

21. Fuglsang E, Pizzolla A, Krych L, Nielsen DS, Brooks AG, et al. (2018) Changes in Gut Microbiota Prior to Influenza A Virus Infection Do Not Affect Immune Responses in Pups or Juvenile Mice. Front Cell Infect Microbiol 8: 319. 


\section{Journal of Infectious Diseases \& Travel Medicine}

22. Hirayama K, Miyaji K, Kawamura S, Itoh K, Takahashi E, et al. (1995) Development of Intestinal Flora of Human-Flora-Associated (Hfa) Mice in the Intestine of Their Offspring. Experimental Animals 44(3): 219. 222.

23. Speshock JL, Doyon-Reale N, Rabah R, Neely MN, Roberts PC (2007) Filamentous influenza A virus infection predisposes mice to fatal septicemia following super infection with Streptococcus pneumoniae serotype 3. Infect Immun 75(6): 31023111.

24. Deriu E, Boxx GM, He X, Pan C, Benavidez SD, et al. (2016) Influenza Virus Affects Intestinal Microbiota and Secondary Salmonella Infection in the Gut through Type I Interferons. PLoS Pathog 12(5): e1005572.

25. Radigan KA, Misharin AV, Chi M, Budinger GS (2015) Modeling human influenza infection in the laboratory. Infect Drug Resist 8: 311-320.

26. Rello J, Pop-Vicas A (2009) Clinical review: primary influenza viral pneumonia. Crit Care 13(6): 235.

27. Bouvier NM, Lowen AC (2010) Animal Models for Influenza Virus Pathogenesis and Transmission. Viruses 2(8): 1530-1563.

28. Sun S, Zhao G, Xiao W, Hu J, Yan Guo, et al. (2011) Age-related sensitivity and pathological differences in infections by 2009 pandemic influenza A (H1N1) virus. Virol J 8: 52.

29. Tate MD, Schilter HC, Brooks AG, Reading PC (2011) Responses of mouse airway epithelial cells and alveolar macrophages to virulent and avirulent strains of influenza a virus. Viral Immunol 24(2): 77 88.

30. Rutigliano JA, Sharma S, Morris MY, Oguin TH 3rd, McClaren JL, et al. (2014) Highly pathological influenza A virus infection is associated with augmented expression of PD-1 by functionally compromised virus-specific CD8+ $\mathrm{T}$ cells. J Virol 88(3): 1636-1651.

31. Job ER, Bottazzi B, Short KR, Deng YM, Mantovani A, et al. (2014). A single amino acid substitution in the hemagglutinin of H3N2 subtype influenza A viruses is associated with resistance to the long pentraxin PTX3 and enhanced virulence in mice. J Immunol 192(1): 271-281.
32. Pyndt Jørgensen B, Hansen JT, Krych L, Larsen C, Klein AB, et al. (2014). A possible link between food and mood: dietary impact on gut microbiota and behavior in BALB/c mice. PLoS One 9(8): e103398.

33. Edgar RC (2013) UPARSE: highly accurate OTU sequences from microbial amplicon reads. Nat Methods 10(10): 996-998.

34. Caporaso JG, Kuczynski J, Stombaugh J, Bittinger K, Bushman FD, et al. (2010) QIIME allows analysis of high-throughput community sequencing data. Nat Methods 7(5): 335-336.

35. Daniel McDonald, Morgan N Price, Julia Goodrich, Eric P Nawrocki, Todd Z DeSantis, et al. (2012) An improved Greengenes taxonomy with explicit ranks for ecological and evolutionary analyses of bacteria and archaea. ISME J 6(3): 610-618.

36. Oksanen J, Blanchet FG, Kindt R, Legendre P, O'Hara RB, et al. (2014) Vegan: Community Ecology Package, 2011. R package version 2.2-0.

37. Mandal S, Van Treuren W, White RA, Eggesbø M, Knight R, et al. (2015) Analysis of composition of microbiomes: a novel method for studying microbial composition. Microb Ecol Health Dis 26: 27663.

38. Desbonnet L, Clarke G, Traplin A, O'Sullivan O, Crispie F, et al. (2015). Gut microbiota depletion from early adolescence in mice: Implications for brain and behaviour. Brain Behav Immun 48: 165-173.

39. Dubos R, Schaedler RW, Costello RL (1963) The Effect of Antibacterial Drugs on the Weight of Mice. J Exp Med 117(2): 245-257.

40. Marx JO, Vudathala D, Murphy L, Rankin S, Hankenson FC (2014) Antibiotic administration in the drinking water of mice. J Am Assoc Lab Anim Sci 53(3): 301306.

41. Derrien $M$, Vaughan EE, Plugge CM, de Vos WM (2004) Akkermansia muciniphila gen. nov., sp nov., a human intestinal mucin-degrading bacterium. International Journal of Systematic and Evolutionary Microbiology 54: 1469-1476.

42. Kim YS, Ho SB (2010) Intestinal goblet cells and mucins in health and disease: recent insights and progress. Curr Gastroenterol Rep 12(5): 319-330. 